\title{
Microturbulence driven transport of energetic ions in the ITER steady-state scenario
}

\author{
M. Albergante, J.P. Graves, A. Fasoli and X. Lapillonne \\ Centre de Recherches en Physique des Plasmas, Association EURATOM-Confédération \\ Suisse, 1015 Lausanne, Switzerland \\ E-mail: mattia.albergante@epfl.ch
}

Received 28 October 2009, accepted for publication 18 January 2010

Published 28 July 2010

Online at stacks.iop.org/NF/50/084013

\begin{abstract}
Modelling of microturbulence-driven transport of energetic ions in an ITER steady-state scenario is presented. Results indicate that a significant fraction of the velocity space distribution of alpha particles and deuterium ions can be transported above neoclassical predictions. Turbulent magnetic fluctuations are found to significantly enhance the fast ion diffusivity. Overall, the conclusion that turbulent fields have a limited influence on the transport of fusion born alpha particles is drawn. At the same time, anomalous fast ion diffusion could have an impact on the neutral beam driven current in ITER.
\end{abstract}

PACS numbers: $52.55 . \mathrm{Pi}$, 52.35.Ra

(Some figures in this article are in colour only in the electronic version)

\section{Introduction}

The physics of energetic ions in tokamak plasmas has been an important area of investigation, both experimentally and theoretically, over the last decades. In thermonuclear plasmas, energetic particles give their energy to the background species via collisional processes until full thermalization. This is the physical mechanism that should keep the plasma temperature sufficiently high to maintain burning plasma conditions. Throughout this process, and in the absence of strong plasma waves, the particles experience a displacement from their equilibrium orbit. The overall result is the radial broadening of the otherwise time-invariant fast ion density profile, whose diffusivity is not expected to be larger than $D_{\text {coll }} \simeq 0.01-$ $0.1 \mathrm{~m}^{2} \mathrm{~s}^{-1}$ in ITER [1].

Collisional estimates can be exceeded whenever interactions with Alvén waves, toroidal field ripple or large scale magnetohydrodynamic (MHD) waves are present [2]. Smallscale turbulence effects, on the other hand, have so far been neglected when studying the transport of energetic ions as the fast gyro-motion causes the particles to experience an 'effective' or averaged turbulent field [3]. A similar mechanism is expected for the drift-orbit motion of energetic ions [4] and experiments consistent with this picture were found in TFTR [5-7].

Recently, the anomalous redistribution of beam ions in the absence of MHD or Alvén mode activity has been observed experimentally. An anomalous diffusion coefficient had to be taken into account in order to explain the anomalous behaviour of the current drive profile in ASDEX Upgrade [8,9]. Since the fast ion redistribution was correlated with the background plasma heat transport, the conclusion that the fast ion transport was caused by microturbulence was drawn. More recently, on the DIII-D tokamak [10], a series of experiments for the development of advanced scenarios were characterized by the unexpected behaviour of energetic ions [11]. This anomaly was particularly interesting due to the absence of modes already known to drive fast ion transport. The introduction of an anomalous diffusion coefficient was needed to improve the agreement between TRANSP calculations and the motional Stark effect (MSE) polarimetry observations. Further investigations were then carried out and a series of experiments with varying plasma temperatures, while keeping the injected fast ion characteristics unaltered, were undertaken [12]. It was shown in this work how the discrepancy between experimental observation and theoretical predictions appeared when considering data from the MSE and the fast ion $\mathrm{D}_{\alpha}$ (FIDA [13]) diagnostics. The disagreement, particularly evident at large temperatures, could be reduced by introducing an anomalous diffusion coefficient from gyrokinetic predictions. The conclusion that microturbulence was responsible for the observed anomaly was therefore drawn. Differences between TRANSP predictions and experimental evidence have also been found at the Joint European Torus 
(JET [14]) during neutral beam injection of tritons. In this case, the loss of a large fraction of passing ions had to be taken into account in order to match the neutron emission in intensity and spatial distribution [15]. The anomalies presented in the latter work were evident only for high density ELMy $\mathrm{H}$-mode plasmas and relatively low values of $q_{95}<4$. The presence of MHD modes with $n=1$ and $n=2$ toroidal numbers was observed during the discharge, although the authors demonstrated that this particular activity could not be linked to the observed fast ion behaviour. In this case, the physical mechanism behind the anomalous redistribution/loss of beam ions is yet to be discovered.

Numerically and theoretically, the anomalous transport of energetic ions has increasingly attracted attention, triggered by the work of Estrada-Mila et al [16] and the previously described experimental evidence. In the work of [16], a series of nonlinear simulations was performed showing that high temperature Maxwellian distributions, representative of slowing-down populations of alpha particles, could be transported well above the background plasma species. Also, the presence of a particle pinch for a particular set of parameters was found, and it was demonstrated that under certain conditions alpha particles could have been transported towards the centre of the plasma. Unfortunately, this particularly favourable condition for burning plasma operation is not likely to occur as it requires the presence of a steep temperature gradient for alpha particles, which, on the contrary, is expected to be fairly flat. In the same work, a quasilinear model was also developed, confirming the nonlinear results obtained with the GYRO code [17]. The larger discrepancy between the quasilinear model and nonlinear simulations was found for the heat diffusivity. This result implicitly demonstrated the inapplicability of quasilinear models when dealing with highly energetic particles which play an important role in the calculation of the heat flux. The inaccuracy of quasilinear models can be seen as a demonstration that nonlinear decorrelation phenomena begin to play a dominant role for this class of particles, and orbit-averaging effects, conventionally considered as very effective in suppressing the turbulent transport, are not valid (see, e.g., the theoretical and numerical analysis of $[18,19])$. In [16], the authors also dealt with an asymptotic theory to simplify the study where quasilinear models could be employed and a particle diffusivity scaling as $D \simeq\left(T_{\mathrm{e}} / T_{\alpha}\right)^{3 / 2}$ was found for the first time. The results of $[9,16]$ captured the attention of the community, and the anomalous transport of fast ions, usually considered a wellunderstood branch of plasma physics, has been extensively investigated since then (see, e.g., [20-26]). The general conclusion from the literature is that anomalous transport can indeed lead to the redistribution of fast ions, especially for high temperature plasmas and for those particles whose energies are of the order of, or even larger than, $10 T_{\mathrm{e}}$. In particular, the transport of passing ions is expected to be the largest, as the relatively long energy tail $D \simeq\left(T_{\mathrm{e}} / E\right)$, where $E$ is the energy of the particle, is expected. This scaling was first discovered in [21], and later found a theoretical explanation in [18, 20]. A different dependence characterizes trapped particles, for which two different energy decays have been proposed, $D \simeq\left(T_{\mathrm{e}} / E\right)^{2}$ or $D \simeq\left(T_{\mathrm{e}} / E\right)^{3 / 2}$, according to $[18,20]$, respectively. In [19] it has also been shown that at non-negligible plasma $\beta$ values anomalous transport driven by magnetic fluctuations can become significant.

In this work, we study both electrostatic and electromagnetic anomalous transport of energetic ions in the ITER steadystate scenario plasma discharge. The analysis is performed by means of the gyrokinetic code GENE [27,28], whose main features are described throughout section 2. Density, temperature and safety factor profiles are taken from transport models in order to appropriately set the parameters for the GENE simulation. The background plasma is modelled as a mixture of deuterium ions and electrons, and only ITG modes at finite plasma $\beta$ are considered. Two suprathermal species describing deuterium and alpha particles are introduced to study the fast ion transport. These particular species are considered as passive tracers, a commonly employed approximation [19, 21, 24]. The fast ions are therefore considered as being highly diluted, and their impact on the evolution of the drift wave has been ignored. A velocity space dependent ('kinetic') diffusivity is defined in section 2. This choice of variables allows for the detailed analysis of the transport features of narrow regions in velocity space. This approach helps better understand which regions of the distribution function of energetic ions are mostly influenced by the turbulent fields. Given the importance of energetic ions in burning plasma conditions, both for the achievement of long pulses and for heating purposes, the model is applied to the ITER steady-state scenario. The results presented in section 3 indicate that electrostatic transport of deuterium ions can significantly exceed neoclassical expectations $\left(0.1 \mathrm{~m}^{2} \mathrm{~s}^{-1}\right)$, as first predicted by [16]. These conclusions are particularly true for particles above the critical energy $\left(E_{\mathrm{c}} \simeq 240 \mathrm{keV}\right)$. Larger transport levels are found for alpha particles, and the neoclassical estimate is exceeded over a wider energy range than for deuterium ions. The possibility that our model overestimated the transport features of the background plasma, and consequently of the fast ions, is considered in section 3.3. Here, we demonstrate that by reducing the strength of the ITG turbulence, the fast ion transport decreases accordingly but still remains significant, at least for NBI ions, and must be included in transport models. Following these considerations, the comparison with previous work, and in particular [16,25], is carried out in section 3.4. The impact of low amplitude magnetic fluctuations is also considered in section 4 . The results are found to be in good agreement with the findings of [19], indicating that the transport of passing particles can be significantly enhanced. In the same section we show that the diffusivity of fast ions is the largest on the low-field side region of the tokamak, where the turbulent fields are the most intense.

\section{The gyrokinetic model}

The turbulent transport of energetic ions is studied throughout the following sections by means of the Eulerian gyrokinetic code GENE [27,28]. The code employs finite difference schemes and 4th order Runge-Kutta methods for the integration of the gyrokinetic Vlasov equation in phase space and time. For our analysis we employ the flux tube version of the code, allowing for a local study of the anomalous transport of fast ions driven by microturbulence. The real space is described by the field-aligned set of coordinates $(x, y, z)$. The 
radial coordinate is described by the variable $x=q_{0}(\psi-$ $\left.\psi_{0}\right) /\left(r_{0} B_{\text {axis }}\right)$, where $\psi$ is the poloidal flux and $q$ is the safety factor. A ' 0 ' subscript indicates a quantity calculated at the centre of the flux tube. The $(y, z)$ variables span over a single flux surface, where $y$ is the binormal coordinate and $z$ defines the position along a field line. The velocity space is described by the variables $\left(v_{\|}, \mu\right)$, with $v_{\|}=\boldsymbol{v} \cdot \hat{\boldsymbol{b}}, \mu=\frac{1}{2} m v_{\perp}^{2} / B$ and $\hat{b}$ the local magnetic field line unit vector. The real plasma geometry is included thanks to the interface [29] with the MHD equilibrium code CHEASE [30]. The GENE code employs a $\delta f$ scheme in order to solve the gyrokinetic Vlasov equation. In its current version, the equilibrium distribution of the $j$ th species is a Maxwellian function

$$
f_{0_{j}} \simeq \frac{n_{j}}{T_{j}^{3 / 2}} \exp \left(-\frac{\frac{1}{2} m_{j} v_{j}^{2}+\mu_{j} B}{T_{j}}\right)
$$

In our study, the background plasma is composed of deuterium ions and electrons, both treated as fully kinetic species. The suprathermal ions are again modelled as Maxwellian distributed, in contrast to the usual assumption of a slowingdown distribution. To reduce the impact of this approximation and remove the dependence for the particular $f_{0}$ employed in the numerical scheme, a set of variables can be defined, as we shall see in the next section. The same procedure allows for a detailed velocity space analysis.

Our study is focused on the radial component of the nonlinear particle flux $\Gamma$, defined as

$$
\Gamma(\boldsymbol{x})=\hat{\boldsymbol{e}}_{r} \cdot \boldsymbol{\Gamma}(\boldsymbol{x})=\hat{\boldsymbol{e}}_{r} \cdot \int \mathrm{d}^{3} v \delta f(\boldsymbol{x}, \boldsymbol{v}) \delta \boldsymbol{u}(\boldsymbol{x}, \boldsymbol{v}) .
$$

Here, $\delta f$ is the perturbation from the initial distribution function $f_{0}, \delta \boldsymbol{u}$ is the perturbed drift of the particle and $\hat{\boldsymbol{e}}_{r}$ is the radial unit vector. A separation between the 'electrostatic' and 'electromagnetic' components of the particle flux is introduced:

$$
\begin{gathered}
\Gamma^{\mathrm{es}}(\boldsymbol{x})=\hat{\boldsymbol{e}}_{r} \cdot \int \mathrm{d}^{3} v \delta f(\boldsymbol{x}, \boldsymbol{v}) \delta \boldsymbol{u}_{E \times B}(\boldsymbol{x}, \boldsymbol{v}), \\
\Gamma^{\mathrm{em}}(\boldsymbol{x})=\hat{\boldsymbol{e}}_{r} \cdot \int \mathrm{d}^{3} v \delta f(\boldsymbol{x}, \boldsymbol{v}) \delta \boldsymbol{u}_{A_{\|}}(\boldsymbol{x}, \boldsymbol{v}) .
\end{gathered}
$$

The perturbed drifts are given by

$$
\begin{gathered}
\delta \boldsymbol{u}_{E \times B}=-\frac{\nabla \delta \bar{\Phi} \times \boldsymbol{B}}{B^{2}}, \\
\delta \boldsymbol{u}_{A_{\|}}=-v_{\|} \frac{\nabla \delta \overline{A_{\|}} \times \boldsymbol{B}}{B^{2}},
\end{gathered}
$$

where $\delta \Phi$ and $\delta A_{\|}$are the perturbed electrostatic and magnetic vector potential, respectively. In our notation, an overbar indicates a gyroaveraged quantity. We note that some of the influence of magnetic perturbations is retained through $\delta f$, which is still calculated by including all the contributions to the perturbed velocity $\delta \boldsymbol{u}$. The particular nomenclature is therefore based on choice of $\delta \boldsymbol{u}$ in (2). In the rest of this work, we shall drop the bold notation whenever referring to scalar quantities obtained by projecting vector quantites along the radial direction.

\subsection{The definition of a kinetic diffusivity}

The nonlinear particle flux defined in $(3 a)$ and $(3 b)$ is strictly related to the particular choice of $f_{0}$ and represents a 'macroscopic' quantity. This definition is well suited for the study of the transport of the background plasma species. On the other hand, energetic ions are characterized by different distribution functions, and a thermal treatment might be inaccurate. The definition of a velocity space dependent ('kinetic') particle flux and diffusivity is therefore needed. We follow the analyses of $[19,26]$ and define the velocity space dependent diffusivity as

$$
D_{v}=-\frac{\Gamma_{v}}{\nabla f_{0}}=-\frac{\delta f}{\nabla f_{0}} \delta u .
$$

Assuming the transport to be diffusive, consistent with the findings of [20], and a Maxwellian unperturbed distribution function, we can simplify (5) as follows:

$$
\begin{gathered}
D_{v}^{\mathrm{es}}=-\frac{1}{\nabla \ln n} \frac{\delta f \delta u_{E \times B}}{f_{0}}, \\
D_{v}^{\mathrm{em}}=-\frac{1}{\nabla \ln n} \frac{\delta f \delta u_{A_{\|}}}{f_{0}} .
\end{gathered}
$$

In this work we study the variable $D_{v}$ defined as the real space and time average of the quantities defined above

$$
D_{v}(\boldsymbol{v})=\left\langle D_{v}(\boldsymbol{x}, \boldsymbol{v}, t)\right\rangle_{x, t} .
$$

Finally, it is interesting to note that a velocity space average of equations ( $6 a)$ and $(6 b)$ over the Maxwellian $f_{0}$ automatically translates into Fick's law

$D \equiv\left\langle D_{v}\right\rangle_{v}=\frac{\int \mathrm{d}^{3} v f_{0} D_{v}}{\int \mathrm{d}^{3} v f_{0}}=-\frac{1}{\nabla \ln n} \frac{\int \mathrm{d}^{3} v \delta f \delta u}{\int \mathrm{d}^{3} v f_{0}}=-\frac{\Gamma}{\nabla n}$,

therefore demonstrating the macroscopic-kinetic correspondence. In the rest of this work, the interpretation of the results is based on the consideration that neoclassical diffusivity of fast ions is not expected to exceed $0.1 \mathrm{~m}^{2} \mathrm{~s}^{-1}$, a value sufficiently high to have an impact on current drive and heat redistribution [9].

\subsection{ITER steady-state scenario}

Steady state will form an important mode of operation in ITER. Based on fully non-inductive driven current, this scenario allows, in particular, for accurate control of the current profile. A fusion gain $Q=5$ is potentially achievable with NBI and ICRH heating techniques. The expected temperature and density profiles, as well as the safety factor and magnetic shear, are shown in figure 1 . We assume electrons and ions to have the same temperature, $T_{\mathrm{e}}=T_{\mathrm{i}}$. The simulation parameters are calculated at $\rho=\sqrt{\psi_{t} / \psi_{t, \text { edge }}}=0.4$, with $n_{\mathrm{e}}=n_{\mathrm{i}}$, $q_{0}=1.55$ and $\hat{s}=(\rho / q) \mathrm{d} q / \mathrm{d} \rho=0.4$. A pessimistic value of $\beta_{\mathrm{e}}=2 \mu_{0} p_{\mathrm{e}_{0}} / B_{\text {axis }}^{2}=0.6 \%$ is employed to avoid the influence of kinetic ballooning modes (KBMs) [31]. At the same radial position we set the logarithmic gradients for electrons and ions to $R_{0} / L_{n_{\mathrm{i}, \mathrm{e}}}=0.3, R_{0} / L_{T_{\mathrm{i}}}=6.4$ and $R_{0} / L_{T_{\mathrm{e}}}=0$, where

$$
\frac{R_{0}}{L_{\chi}}=\frac{R_{0}}{\chi} \frac{\mathrm{d} \chi}{\mathrm{d} x},
$$



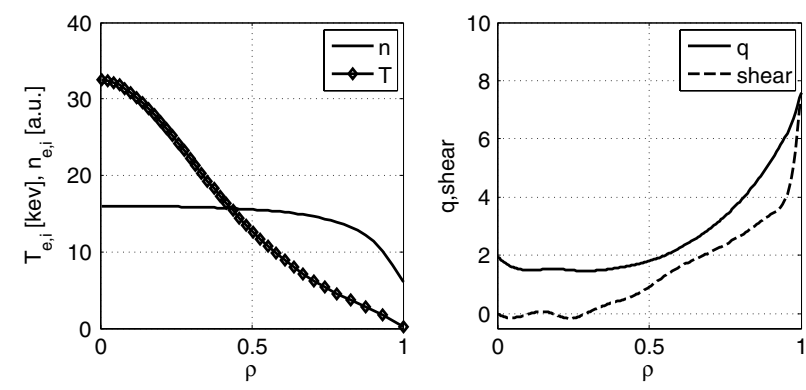

Figure 1. Density and temperature profiles (left) for the background species of the ITER steady-state scenario. On the right, the safety factor and the magnetic shear are plotted.

where $R_{0}$ is the major radius of the tokamak and $x$ is the radial coordinate. The choice of a flat electron temperature profile restricts our analysis to ITG modes only.

The high energy species are treated as passive tracers, i.e. not influencing the space and time evolution of the instability due to their low concentration in the plasma. Two suprathermal species, describing high energy deuterium and alpha particles, are introduced in the simulation. For both energetic species we set the logarithmic gradients to $R_{0} / L_{n}=$ $15, R_{0} / L_{T}=1$ and $T=30 T_{\mathrm{e}}$. These parameters are very different from those of the background plasma and representative of species with a large energy content, strong density gradient and a flat temperature profile, which is usually the case for energetic ions. The definition of $D_{v}$ given in (5) is introduced to remove the dependence from the initial distribution function $f_{0}$ employed in the numerical scheme. The temperature of the passive species is therefore arbitrary and chosen large to cover a sufficiently extended domain in velocity space. For the deuterium population, the velocity space region at large parallel energies is representative of NBI ions, injected at $1 \mathrm{MeV}$ in ITER. We can also draw some general conclusions on the transport of ICRH heated populations, mainly characterized by ions with $\mu B / E \simeq 1$.

\section{Electrostatic component of the particle transport}

Following the description of the physical parameters given in the previous section, we now describe the numerical parameters and the features characterizing the background turbulent fields. The nonlinear GENE simulation is run with a real space resolution of $\left(n_{x}, n_{k_{y}}, n_{z}\right)=(128,22,48)$ points, for a radial extension of $L_{x}=150 \rho_{\mathrm{s}}$ and a maximum resolved wave number of $k_{\perp} \rho_{\mathrm{s}}=\sqrt{g^{y y}} k_{y} \rho_{\mathrm{s}}=1.55$, being $c_{\mathrm{s}}=\sqrt{T_{\mathrm{e}} / m_{\mathrm{i}}}, \rho_{\mathrm{s}}=c_{\mathrm{s}} / \Omega_{\mathrm{i}}$ and $\Omega_{\mathrm{i}}=q_{\mathrm{i}} B / m_{\mathrm{i}}$. The analysis of the linear phase of the instability reveals a dominant ITG mode peaking at $k_{\perp} \rho_{\mathrm{s}}=0.35$, where $\gamma=0.2 c_{\mathrm{s}} / R_{0}$. During the saturated phase of the simulation, the maximum amplitude of the electrostatic field has been found at $k_{\perp} \rho_{\mathrm{s}}=0.21$. A larger scale length, together with a lower intensity, characterizes $\delta A_{\|}$, whose largest amplitude mode is found at $k_{\perp} \rho_{\mathrm{s}} \simeq 0.1$. This feature is evident from figure 2 , where the perturbed fields are represented on real space coordinates. The difference in the mode structure translates into a different importance of gyroaveraging effects.

\subsection{Electrostatic transport of high energy deuterium}

The electrostatic diffusivity of energetic deuterium ions can be seen in figure 3 . This figure is obtained by slicing the radial electrostatic diffusivity $D_{v}^{\mathrm{es}}\left(v_{\|}, v_{\perp}\right)$ of equation $(6 a)$ at different energies, while keeping the ratio $\mu B_{\text {axis }} / E$ constant. We choose the value of $\mu B_{\text {axis }} / E=0.8$ for trapped particles and $\mu B_{\text {axis }} / E=0.4$ for passing particles. The trapped/passing boundary is found at $\mu B_{\text {axis }} / E \simeq 0.75$. As we can see from the curves shown in the figure, a substantial transport of energetic ions is to be expected. The results are in good agreement with the energy scalings described in $[18,19]$, hereby shown as dashed lines. We expect the high energy radial diffusivity of trapped particles to decay as $D_{v}^{\text {es }} \simeq E^{-3 / 2}$ and that of passing ions as $D_{v}^{\mathrm{es}} \simeq E^{-1}$. The faster scaling for trapped particles is due to the presence of gyroaveraging effects. Our results confirm these scalings, showing that both classes of particles experience an enhancement in the radial diffusivity up to $1 \mathrm{MeV}$. It is evident from the figure that the particles in the lower energy range interact significantly with the turbulent fields and that the energy decay proposed in previous work appears only in the high energy limit. This is due to resonant interactions between the characteristic frequencies of the drift-orbit motion of energetic particles and the turbulent field frequency [18]. Let us calculate the resonant condition between the toroidal precession drift of trapped particles [19]

$$
v_{y} \sim(1-\eta)^{2}\left(\frac{E}{T_{\mathrm{e}}}\right) \frac{\rho_{\mathrm{s}} c_{\mathrm{s}}}{R_{0}},
$$

where $\eta=v_{\|} / v$, and the drift wave velocity along the $y$ direction as

$$
v_{\mathrm{dr}}=\frac{\omega}{k_{y}} \simeq 1.5 \frac{\rho_{\mathrm{s}} c_{\mathrm{s}}}{R_{0}},
$$

$\omega$ being the frequency of the turbulent mode. Given our nominal parameters, the resonant interaction is expected at $E \simeq 4.5 T_{\mathrm{e}}$, which is approximately $70 \mathrm{keV}$ for the scenario of interest. This estimate is outside the energy range of our simulation and further analysis will be carried out to verify the position of the interaction peak. Nevertheless, it is important to underline the presence of this resonance as it keeps the particle diffusivity sufficiently high over a wide energy range. As a result, the transport of particles at moderate energies $\left(1<E / T_{\mathrm{e}}<10\right)$ can be comparable to the background plasma transport coefficients. At larger energies the transport is smaller and its magnitude is both influenced by the position of the resonant peak, and the class of particles considered (whether trapped or passing).

\subsection{Electrostatic transport of alpha particles}

The anomalous transport of alpha particles is characterized by results similar to those obtained for the high energy deuterium ions. The curves in figure 4 clearly show that enhanced diffusivities can be found over a very large energy range (up to $E=3 \mathrm{MeV}$ ) for both passing and trapped particles. The shape in the diffusivity of alpha particles is very similar to that of deuterium ions, with a shift towards higher energies due to a heavier mass. Therefore, we find a region at moderate energies characterized by a slowly decaying diffusivity. These results once again underline the importance of the low energy 



Figure 2. Snapshot of the perturbed electrostatic (left) and magnetic (right) potential in the ITER steady-state scenario. (Colour online.)


Figure 3. Electrostatic particle diffusivity of high energy deuterium in the ITER steady-state scenario, as a function of energy. The numerical results for passing ions $\left(\mu B_{\text {axis }} / E=0.4\right.$, left panel) and trapped ions ( $\mu B_{\text {axis }} / E=0.8$, right panel) are plotted together with the scalings predicted by [19] (dashed lines). These are, namely, $D_{v}^{\text {es }} \simeq E^{-1}$ for passing particles and $D_{v}^{\text {es }} \simeq E^{-3 / 2}$ for trapped particles.
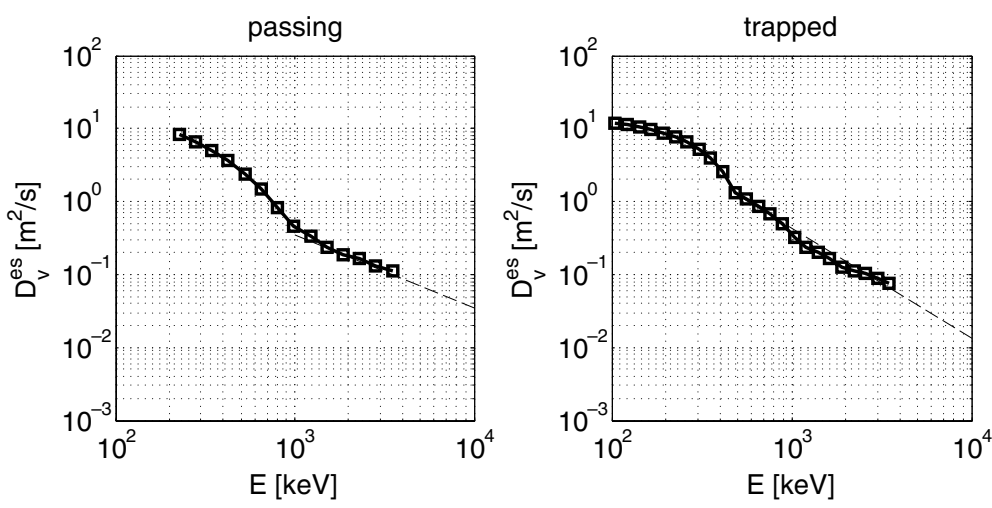

Figure 4. Electrostatic particle diffusivity $D_{v}^{\text {es }}$, equation (6a), of passing $\left(\mu B_{\text {axis }} / E=0.4\right.$, left panel) and trapped $\left(\mu B_{\text {axis }} / E=0.8\right.$, right panel) alpha particles as a function of energy. The theoretical scalings of $[18,19], D_{v}^{\text {es }} \simeq E^{-1}$ for passing particles and $D_{v}^{\text {es }} \simeq E^{-3 / 2}$ for trapped particles, are also plotted (dashed lines).

resonance, as this resonance is shifted towards higher energies for alpha particles. This explains the higher diffusivity obtained with respect to the deuterium case. For this reason, the energy scalings of $[18,19]$ appear at larger energies than for deuterium ions. The results shown here indicate that the radial transport of alpha particles can significantly exceed neoclassical predictions. Together with the results of the previous section, we can also observe that the ${ }^{3} \mathrm{He}$ population, subject to ICRH heating in ITER steady-state operation, is also expected to be transported above neoclassical levels. The results demonstrate that plasma heating and current drive techniques will potentially be affected by fast ion redistribution in ITER, which is only in apparent contradiction with the conclusions of [25] as we shall see in the next sections.

\subsection{Effect of a reduced instability drive}

Although the anomalous transport of energetic ions has been demonstrated as being important in the previous section, the heat conductivity of the background ions is $\chi_{\mathrm{i}} \simeq 15 \mathrm{~m}^{2} \mathrm{~s}^{-1}$. This is a large value if compared with the expected anomalous heat conductivity which is of the order of $1 \mathrm{~m}^{2} \mathrm{~s}^{-1}$. A possible explanation for the large diffusivity is that the instability drive can be too large once introduced in the gyrokinetic 

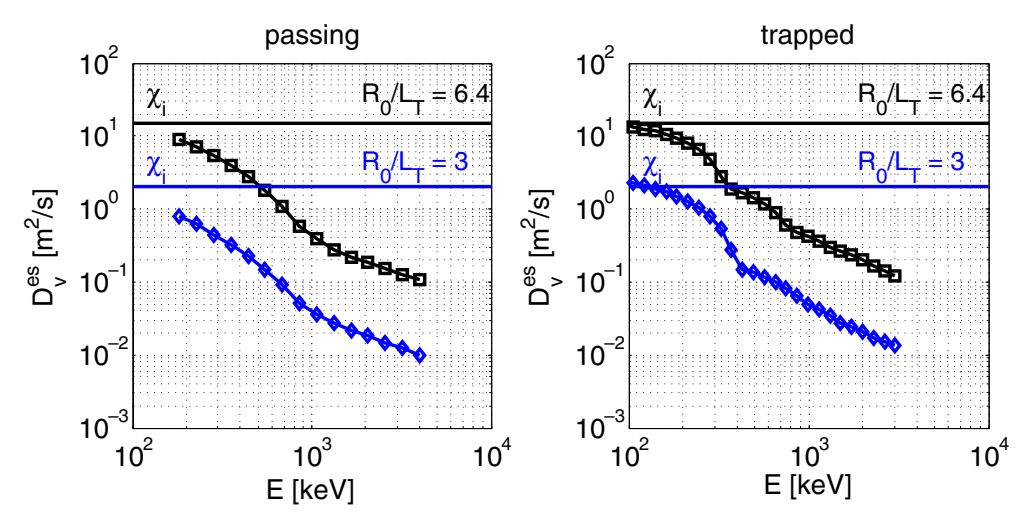

Figure 5. Electrostatic particle diffusivity $D_{v}^{\text {es }}$ of alpha particles, as a function of energy, for passing ions ( $\mu B_{\text {axis }} / E=0.4$, left panel) and trapped ions $\left(\mu B_{\text {axis }} / E=0.8\right.$, right panel). The numerical results of the simulation with a reduced instability drive $\left(R_{0} / L_{T_{i}}=3\right.$, diamonds $)$ are plotted together with the results of the original ITER simulation $\left(R_{0} / L_{T_{i}}=6.4\right.$, squares). (Colour online.)

model. Moreover, the flux tube version of the GENE code, hereby employed, does not allow for profile relaxation. The simulation presented throughout this work could therefore represent a plasma scenario far from marginal stability, as already discussed in [16]. To estimate the fast ion transport in a plasma characterized by a more realistic transport of the background species, and consequently by a less strong ITG turbulence, we can artificially reduce the instability drive. The ion temperature gradient length has been decreased from $R_{0} / L_{T_{\mathrm{i}}}=6.4$ to $R_{0} / L_{T_{\mathrm{i}}}=3$ in order to reach a background plasma heat diffusivity of $\chi_{\mathrm{i}} \simeq 2 \mathrm{~m}^{2} \mathrm{~s}^{-1}$. We then apply the same numerical technique of the previous sections and we obtain, for alpha particles, the results plotted in figure 5. We notice a remarkable decrease in the transport of the energetic species, which occurs as a result of the reduction in background conductivity due to the change in $R_{0} / L_{T_{\mathrm{i}}}$. We can clearly see how the energetic ions can, nevertheless, be significantly transported, although on energy ranges smaller with respect to previous case. With this choice of parameters, only alpha particles below $600-700 \mathrm{keV}$ suffer from anomalous transport sufficiently large to importantly modify their trajectory. As a result, the slowing-down process would still take place and fusion born alphas could give most of their energy to the background species before experiencing the turbulent fields. In the next section, we will see how similar conclusions cannot be drawn for the NBI population.

\subsection{Comparison with previous work}

Let us now compare the results obtained in the previous sections with previous work, especially the electrostatic findings of Estrada-Mila [16] and Angioni [25]. The study of the magnetic component of the particle transport will be studied in a separate section. In the analysis of [16], the transport of Maxwellian distributions of helium impurities at varying temperature was studied. The particle diffusivity of populations characterized by a large temperature was then associated with the transport of alpha particles. To compare our results with those presented in [16], we can solve equation (8) for an unperturbed Maxwellian $f_{0}$, at increasing temperature. The particle diffusivity $D(E)$ of equation (8) can be defined according to the results of our work. Therefore, we assume the diffusivity to be constant for particles at energies smaller than an arbitrary energy $E=L T_{\mathrm{e}}$. This approximation is based on the observation of section 3.1. At larger energies, nonlinear decorrelation phenomena become important and the energy decay follows a power law $D \simeq\left(T_{\mathrm{e}} / E\right)^{n}$,

$$
D / \chi_{b g}= \begin{cases}1 & \text { if } E \leqslant L T_{\mathrm{e}}, \\ {\left[T_{\mathrm{e}} /(L E)\right]^{n}} & \text { elsewhere. }\end{cases}
$$

We choose parameters consistent with the results presented throughout this work, $L=4$ and $n=1.2$ (predominantly passing particles). The results obtained with this procedure, presented in figure 6 , can finally be compared with the nonlinear results presented in figure 8 of [16]. The similarity between the findings is evident. In figure 6 we can also observe the diffusivity decay at high temperatures, which follows the power law $D \simeq\left(T_{\mathrm{e}} / T_{\alpha}\right)$. This scaling can also be obtained by analytically solving equation (8), which can be written in this case as

$$
D\left(T_{\alpha}\right)=\frac{\int \mathrm{d} E \sqrt{E} D(E) \mathrm{e}^{-E / T_{\alpha}}}{\int \mathrm{d} E \sqrt{E} \mathrm{e}^{-E / T_{\alpha}}} .
$$

To ease the calculation, we shall employ $n=1$ in the definition of $D(E)$ as from equation (12). Defining $\lambda_{\alpha}=T_{\alpha} / T_{\mathrm{e}}$, we obtain

$$
\begin{aligned}
D\left(\lambda_{\alpha}\right)= & -\sqrt{L / \lambda_{\alpha}} \mathrm{e}^{-L \lambda_{\alpha}}+\Gamma\left(\sqrt{L / \lambda_{\alpha}}\right) \\
& \times\left(\frac{1}{2} \sqrt{\pi}-L \sqrt{\pi} / \lambda_{\alpha}\right)+L \sqrt{\pi} / \lambda_{\alpha},
\end{aligned}
$$

where $\Gamma$ is the error function. The first-order Taylor expansion of the previous expression for $\lambda_{\alpha} \rightarrow \infty$ gives the expected $D \simeq \lambda_{\alpha}^{-1}$ dominant term. In [16], an asymptotic analysis revealed the existence of the slightly faster scaling $D \simeq \lambda_{\alpha}^{-3 / 2}$. The different theoretical prediction of [16] could be justified either by the particular approximations introduced in the latter work (large shear, no parallel dynamics) or by the simple model defined in equation (12). Also, the linear model could be inaccurately describing the long energy tails in the diffusivity, which is a nonlinear phenomenon. The difference between linear and nonlinear theories, however, is probably insignificant when describing distributions characterized by fast energy tails (i.e. thermal populations), as already pointed out in $[16,25]$. The difference between the two models could, nevertheless, emerge when considering the heat diffusivity, which is importantly influenced by the high energy characteristics of $D(E)$. 

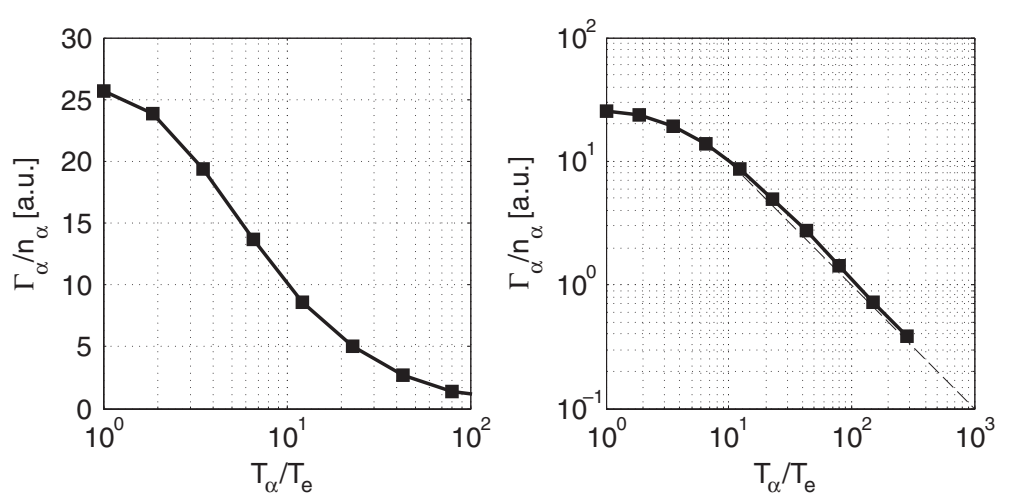

Figure 6. Particle diffusivity for a Maxwellian population of alpha particles, as a function of the temperature of the distribution (left and right panels). An arbitrary normalization has been included in the curve to simplify the comparison with the results presented in [16]. The right panel shows the same data, but plotted in log-log scale, in order to demonstrate the $\Gamma \simeq\left(T_{\mathrm{e}} / T_{\alpha}\right)$ dependence and eventual power-law (dashed line).

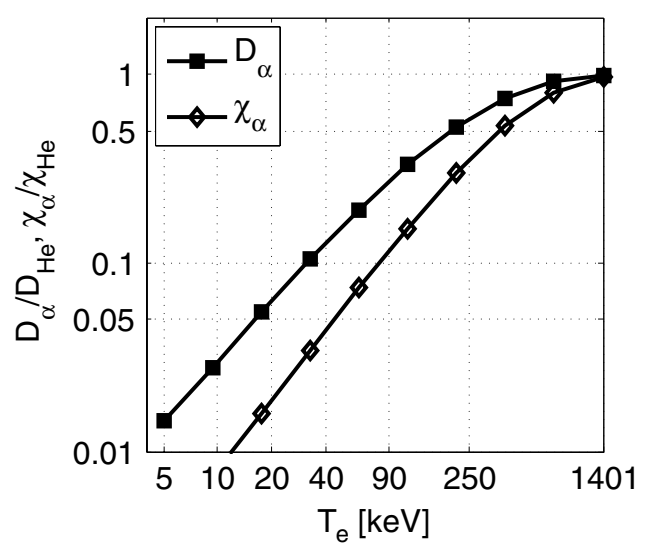

Figure 7. Particle and heat diffusivity for a Maxwellian population of alpha particles, as a function of the plasma temperature. This figure underlines the good agreement with the results of figure 7 in a recent work by Angioni [25].

The scaling law $D \simeq \lambda_{\alpha}^{-1}$ has also been found by Angioni and co-workers. For example, this scaling can be inferred from figure 6 of [25]. Further similarities with [25] can be found by solving equation (8) for both particle and heat diffusivities of Maxwellian distributions. By applying the normalization employed in [25], we obtain the results of figure 7, whose resemblance to figure 6 of [25] is noticeable. Also investigated in [25] was the electrostatic transport of alpha particles modelled as a slowing-down population and implemented in the GS2 code [32]. The anomalous particle transport obtained by means of nonlinear gyrokinetic simulations was then implemented in the ASTRA code for evaluating the impact of microturbulence on the alpha particle density profile. The conclusion that electrostatic turbulence has a negligible effect on the transport of alpha particles was eventually drawn. By employing an unperturbed slowing-down distribution function of alpha particles in equation (8), we can show that the contradiction between the conclusions of [25] and those of our work is only apparent. For a realistic slowing-down distribution of alpha particles in ITER, we obtain $D_{\alpha} / \chi_{b g}=$ 0.07 which brings to an absolute value of $D_{\alpha} \simeq 0.15 \mathrm{~m}^{2} \mathrm{~s}^{-1}$, a value larger than neoclassical predictions, but nevertheless small. The reason for this relatively small estimate comes from the weighting function $f_{0}$, which has long energy tails and therefore selects the very low diffusivity of energetic particles. As a consequence, the transport of a slowingdown distribution of alpha particle is negligible, as concluded in [25]. Nevertheless, those populations characterized by a lower birth energy and a larger passing fraction, such as for an NBI distribution, could still be significantly transported. For the NBI population the expected diffusivity would be $D_{n b i} / \chi_{b g}=0.24$, or $D \simeq 0.5 \mathrm{~m}^{2} \mathrm{~s}^{-1}$, and hence very significant. Moreover, the model described throughout this section only accounts for electrostatic diffusivities, while magnetic perturbations can have even a larger impact as we shall demonstrate in the next section.

\section{Magnetic component of the particle transport}

As pointed out in [19], the transport of energetic ions in the presence of magnetic perturbations can be higher than the electrostatic equivalent, especially for high energy passing particles. By looking at the perturbed drifts defined in $(4 a)$ and $(4 b)$, it is clear how the proportionality between the parallel velocity of a particle and the perturbed magnetic drift $\delta u_{A_{\|}}$ can drive strong diffusivities for high energy particles despite having low amplitude perturbations

$$
\frac{\delta B_{\perp}}{B} \simeq \frac{k_{\perp} \delta A_{\|}}{B_{\text {axis }}}<10^{-4} .
$$

To verify this assumption, we consider the finite $\beta$ simulation described at the beginning of section 2.2 and the definition of a 'magnetic' diffusivity given in $(6 b)$. We fix a low value of $\mu B_{\text {axis }} / E=0.1$ and increase the energy to study the effect of the perturbed drift on the diffusion coefficient of passing particles. For studying the effect of magnetic fluctuations on trapped particles, we perform a slice of $D_{v}^{\mathrm{em}}$ at constant $\mu B_{\text {axis }} / E=0.9$ and increasing energy. The results for both passive species are plotted in figure 8 . It is observed how the magnetic transport of energetic ions can be significant (around $0.2 \mathrm{~m}^{2} \mathrm{~s}^{-1}$ ) for particles with large parallel energies. The deviation from the analytical expectations seems to be limited to passing alpha particles only. A possible inconsistency with previous theoretical work is that the diffusivity as defined in our work can become negative. 

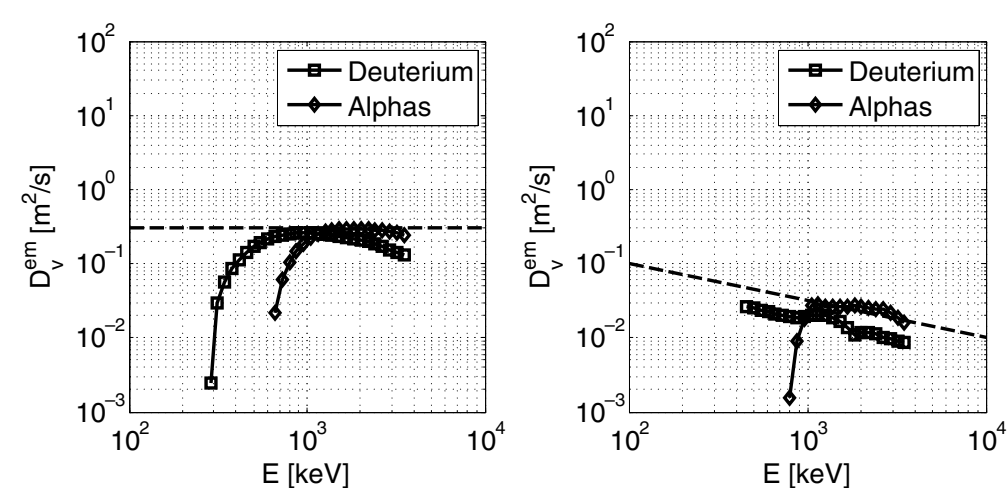

Figure 8. Magnetic particle diffusivity of alpha particles (diamonds) and high energy deuterium (squares) in the ITER steady-state scenario, as a function of energy. The numerical results for passing particles $\left(\mu B_{\text {axis }} / E=0.1\right.$, top left panel) and trapped ions $\left(\mu B_{\text {axis }} / E=0.9\right.$, top right panel) are plotted together with the scalings predicted by [19] (dashed lines). These are, namely, constant diffusivity for passing particles and $D_{v}^{\mathrm{em}} \simeq E^{-1 / 2}$ for trapped particles.

In [19] (8) the diffusivity is defined as a quadratic function of the particle velocity and negative values are not allowed in this framework. However, negative values are found in our work for low energy regions and might indicate that ions are not yet decorrelated, and that different physical mechanisms are responsible for the transport. Moreover, our analysis relies on the definition of an effective diffusivity. In the lower energy range of the distribution, non-diffusive processes (convection and thermodiffusion [16]) might be dominant and drive negative fluxes.

The particular case of magnetic transport demonstrates the importance of a velocity space resolved analysis if compared with a Maxwellian case. If we consider the definition of equation (8) and we average the particle flux over a Maxwellian function $f_{0}$, the magnetic diffusivity of a large temperature distribution would become negative. This is due to having most of the particles in the low energy range of the distribution and the global transport features are mostly influenced by this energy region. Therefore, the transport features of high energy particles are completely hidden by employing a macroscopic approach.

It should be noted that the simulations performed in this paper use a $\beta_{\mathrm{e}}$ value smaller than expected $(\simeq 1 \%)$. Increasing $\beta_{\mathrm{e}}$ will generally bring a slight reduction in the electrostatic anomalous transport, both for the background species and suprathermal particles (see, e.g., the results of [31]). At the same time, the perturbed vector potential amplitude increases accordingly, therefore enhancing the magnetic transport described throughout this section. Overall, we can conclude that under certain circumstances and for particular classes of particles, magnetic transport not only can be more significant than neoclassical expectations but can also exceed anomalous electrostatic transport.

\subsection{Poloidal dependence of the particle diffusivity}

Throughout the previous sections, a real space average of the particle diffusivity has been employed in the velocity space investigation of the fast ion transport. This average has then been performed in order to simplify the analysis and is valid so long as a certain degree of symmetry in real space is present. The structure of the turbulent fields, however, can also present asymmetries, or large structures, in real space.
These asymmetries can be more enhanced radially, such as in the presence of blobs and zonal flows, or in the poloidal direction. The latter asymmetry is observable in the presence of drift waves, where the perturbed fields are the most intense in the low-field side region of the tokamak. We now want to estimate to what level the asymmetry in the turbulent fields might also be present in the radial diffusion coefficient. We therefore calculate the variable

$$
D_{v}(z)=\left\langle D_{v}\left(\boldsymbol{x}, v_{\|}, \mu\right)\right\rangle_{x, y},
$$

while keeping energy and magnetic moment constant. For this analysis we choose an $800 \mathrm{keV}$ alpha particle at $\mu B_{\text {axis }} / E=$ 0.25 such that the particle is untrapped (passing). A deuterium particle at $500 \mathrm{keV}$, and with the same $\mu B_{\text {axis }} / E$ ratio, is also considered. The radial diffusion coefficient as a function of the poloidal angle is shown in figure 9. As expected, the anomalous transport of alpha particles and deuterium ions is highly dependent on their poloidal position. As a general remark, the electrostatic transport is largely driven in the low-field side region of the plasma. The latter effect is caused by the presence of more intense turbulent fields in this region, as previously anticipated. In the case of the magnetic transport, the particle diffusivity as a function of the poloidal angle exhibits a more elaborated structure with respect to the electrostatic case. This is caused by a complex poloidal structure of the perturbed magnetic vector, although qualitatively the strongest transport is again observed in the so-called bad curvature region at $\theta=0$.

These results, together with the findings of the previous section, clearly point to the conclusion that accurate models need to be developed in order to estimate the impact of turbulent transport on fast ions in ITER. As shown throughout this work, the anomalous diffusion coefficient of these particles strongly depends on the poloidal position of the particle, as well as on its velocity space characteristic. In a recent publication [33], it was shown how a possible interface between gyrokinetic modelling and transport codes can exploit the above-mentioned dependences. In the latter work, the impact that anomalous diffusion could have on fast ion ripple losses in ITER was evaluated. The novelty of the analysis lies in the implementation of an energy and pitch angle dependent anomalous diffusion coefficient in the ASCOT code [34]. The results of [33], indicating that no enhancement in the wall 

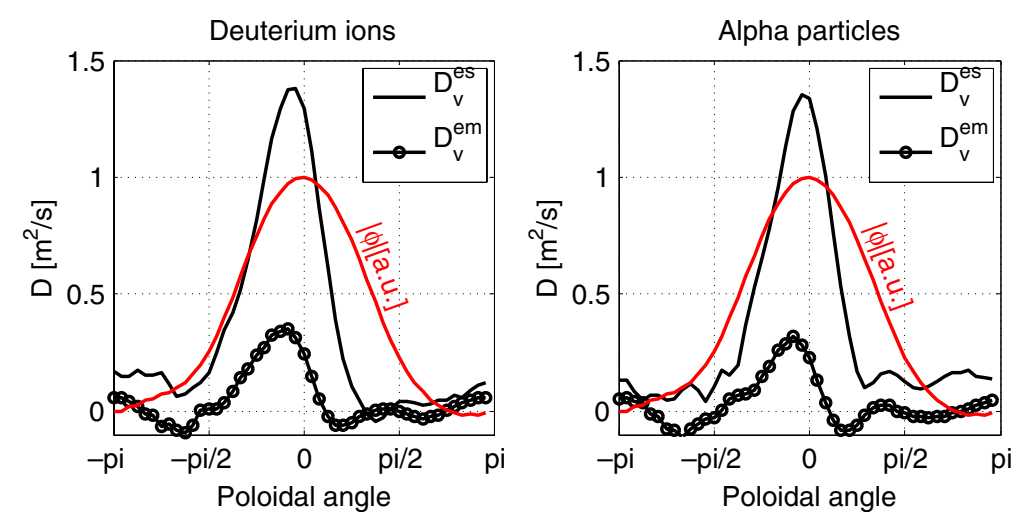

Figure 9. Electrostatic (solid line) and magnetic (circles) particle diffusivity of a $800 \mathrm{keV}$ alpha particle (left panel) and a $500 \mathrm{keV}$ deuterium ion (right panel) as a function of the poloidal angle. Both the particles are characterized by $\mu B_{\text {axis }} / E=0.25$. The electrostatic field intensity as a function of the poloidal angle is also shown. (Colour online.)

loads is to be expected for the parameters employed in the simulation, represent a very promising example and similar analyses must be carried out to assess potential current heat redistribution/losses. The neutral beam driven current also needs to be investigated in the presence of microturbulent fields. This aspect is particularly important as the accurate control of the safety factor profile is a fundamental requirement for steady-state operation and potential degradations must be assessed. In conclusion, accurate models, such as the fully kinetic approach of [33], are needed to verity if, and to what extent, microturbulence could play a role in restraining ITER potential.

\section{Summary}

In this work we have studied the transport of suprathermal particles in the presence of small-scale turbulent fields. Nonlinear gyrokinetic simulations of the ITER steady-state scenario have been performed using the GENE code. The interface with the MHD equilibrium code CHEASE has allowed for the full treatment of the metric coefficients in the gyrokinetic Vlasov equation. The background plasma profiles and the magnetic field structure expected for the ITER steady-state scenario have been employed. Two suprathermal populations, describing energetic deuterium and alpha particles, have then been introduced in the calculation. These two species have been treated as passive tracers, an approximation valid so long as the minority species concentration in the plasma is low. The transport of the passive populations has been studied in detail by introducing a set of velocity dependent ('kinetic') variables. By defining a kinetic diffusivity, in particular, we have been able to investigate more precisely the anomalous transport of energetic ions than by merely studying integrated quantities such as the macroscopic particle diffusivity.

The results indicate that the electrostatic transport of high energy deuterium and alpha particles can be significantly higher than neoclassical estimates. The latter species, in particular, exhibits radial diffusivities above $0.1 \mathrm{~m}^{2} \mathrm{~s}^{-1}$ for energies up to $3 \mathrm{MeV}$. Similar results have been obtained for suprathermal deuterium, although for this species the electrostatic transport becomes negligible above $1 \mathrm{MeV}$. The explanation for such large diffusivities is the lack of drift-orbitaveraging effects for particles whose energies largely exceed the plasma temperature [19]. Similar levels of transport can be found for passing and trapped particles.

Since the latter results are based on simulations characterized by a relatively large background plasma heat conductivity, further analysis has been carried out. By identifying a set of parameters resulting in a heat conductivity closer to ITER expectations $\left(\chi_{\mathrm{i}} \simeq 2 \mathrm{~m}^{2} \mathrm{~s}^{-1}\right)$, the anomalous diffusion of fast ions in a scenario at a weaker level of turbulence has been studied. Results indicate that the transport of alpha particles above $600-700 \mathrm{keV}$, despite being above neoclassical expectation, could nevertheless have only minor consequences. Similar diffusivities are observed for energetic deuterium and, given the lower birth energy for these particles, the neutral beam driven current could still be affected by the presence of small-scale turbulence. These results have then been compared with electrostatic simulations of previous work, where similar findings have been obtained.

The effect of magnetic fluctuations on the transport of suprathermal ions has also been considered in our study and the following conclusions, both for deuterium and helium ions, have been drawn. The magnetic transport of energetic trapped ions is negligible, the main reason being that perturbed electromagnetic drifts are proportional to the parallel velocity of the particles, usually low for this class of particles. At the same time, however, high energy passing particles can be transported not only above neoclassical expectations but also above electrostatic estimates. This occurs due to the relatively large scale length of perturbations in the magnetic vector potential. For this reason, gyroaveraging effects are less effective in suppressing the magnetic transport at intermediate Larmor radius. Finally, it has also been demonstrated that the anomalous transport of energetic ions strongly depends on the poloidal position of the particle. This result has been found both for the electrostatic and the magnetic components of the turbulent transport.

The results presented throughout this work suggest that modelling of burning plasma operations should take into account the potential influence of microturbulent fields. In particular, calculations on the heat deposition, as well as on the neutral beam driven current, should account for possible anomalous redistributions. Also, although anomalous 
transport phenomena can still allow for the full slowing down of fast ions in ITER, small fractions of energetic particles can be expelled from the plasma. The overall consequence of this could be the enhancement of heat load on in-vessel components that can affect ITER operation. Further analyses are needed in order to assess these conclusions, and to provide an accurate description of the transport features of ITER operation.

Euratom @ 2010.

\section{Acknowledgments}

The authors gratefully acknowledge fruitful discussion with F. Jenko. This work was partly supported by the Swiss National Science Foundation. The simulations have been run on the Pleiades 2 cluster at the Ecole Polytechnique Fédérale de Lausanne.

\section{References}

[1] ITER Physics Expert Group on Energetic Particles, Heating and Current Drive and ITER Physics Basis Editors 1999 Nucl. Fusion 392471

[2] Fasoli A. et al 2007 Progress in the ITER Physics Basis chapter 5: Physics of energetic ions 2007 Nucl. Fusion 47 S 264

[3] Manfredi G. et al 1997 Phys. Plasmas 4628

[4] Mynick H. et al 1979 Phys. Rev. Lett. 431506

[5] Heidbrink W.W. et al 1991 Phys. Fluids B 33167
[6] Ruskov E. et al 1991 Nucl. Fusion 122219

[7] Ruskov E. et al 1995 Nucl. Fusion 351099

[8] Herrmann A. et al 2003 Fusion Sci. Technol. 44569

[9] Günter S. et al 2007 Nucl. Fusion 47920

[10] Luxon J.L. 2002 Nucl. Fusion 42614

[11] Murakami M. et al 2009 Nucl. Fusion 49065031

[12] Heidbrink W. et al 2009 Phys. Rev. Lett. 103175001

[13] Van Zeeland M. et al 2009 Plasma Phys. Control. Fusion 51055001

[14] Keilhacker M. et al 2001 Nucl. Fusion 411925

[15] Baranov Y.F. et al 2009 Plasma Phys. Control. Fusion 5144004

[16] Estrada-Mila C. et al 2006 Phys. Plasmas 13112303

[17] Candy J. et al 2003 Phys. Rev. Lett. 91045001

[18] Hauff T. et al 2008 Phys. Plasmas 15112307

[19] Hauff T. et al 2009 Phys. Rev. Lett. 102075004

[20] Zhang W. et al 2008 Phys. Rev. Lett. 101095001

[21] Dannert T. et al 2008 Phys. Plasmas 15062508

[22] Hauff T. et al 2006 Phys. Plasmas 13102309

[23] Hauff T. et al 2007 Phys. Plasmas 14092301

[24] Angioni C. et al 2008 Phys. Plasmas 15052307

[25] Angioni C. et al 2009 Nucl. Fusion 49055013

[26] Albergante M. et al 2009 Phys. Plasmas 16112301

[27] Jenko F. et al 2000 Phys. Plasmas 71904

[28] Dannert T. et al 2005 Phys. Plasmas 12072309

[29] Lapillonne X. et al 2009 Phys. Plasmas 16032308

[30] Lütjens H. et al 1996 Comput. Phys. Commun. 97219

[31] Pueschel M.J. et al 2008 Phys. Plasmas 15102310

[32] Kotschenreuther M. et al 1995 Comput. Phys. Commun. 88128

[33] Kurki-Suonio T. et al Nucl. Fusion submitted

[34] Kurki-Suonio T. et al 2009 Nucl. Fusion 49095001 